\title{
El maternés de madres peruanas: Una descripción psicolingüística sobre los estilos de habla ${ }^{1}$
}

\section{The Motherese of Peruvian Mothers: A Psycholinguistic Description of Speech Styles}

\author{
Marco Antonio Lovón Cueva \\ Universidad Nacional Mayor de San Marcos, Lima, Perú \\ Grupo de Investigación Lenguas y Filosofía del Perú \\ https://orcid.org/oooo-0oo2-9182-6072 \\ mlovonc@unmsm.edu.pe \\ Arturo Martel Paredes \\ Universidad Nacional Mayor de San Marcos, Lima, Perú \\ Grupo de Investigación Lenguas y Filosofía del Perú \\ https://orcid.org/oooo-0oo1-5408-6215 \\ arturomartelp@gmail.com \\ Melissa Tejada Tirado \\ Pontificia Universidad Católica del Perú \\ a20065148@pucp.edu.pe
}

\begin{abstract}
Resumen
Las madres suelen hablar a sus hijos desde temprana edad para estimular sus habilidades comunicativas y el aprendizaje de su lengua materna. Esta forma especial de comunicación que se desarrolla entre madres (y cuidadores en general) y niños se denomina maternés. En el proceso de adquisición lingüística, el maternés juega un rol central como proveedor de estímulos lingüísticos. El objetivo de este estudio es analizar el maternés de madres peruanas. Metodológicamente, se han seleccionado dos madres y sus niños en la temprana infancia, a quienes se les grabó sus interacciones verbales. En este estudio, se describen las características lingüísticas y los estilos del maternés de estas madres. Asimismo, se da a conocer la diferencia de habla entre la madre de estilo receptivo y la madre de estilo poco receptivo.
\end{abstract}

Palabras claves: maternés, adquisición del lenguaje, castellano, estilo, comunicación.

1 Este estudio se llevó a cabo en el año 2008, como parte de una pesquisa psicolingüística sobre la comunicación madre-hijo, con el fin de analizar la adquisición de una lengua por parte de los niños en interacciones sociales. Agradecemos los alcances brindados en su momento por la lingüista Cecilia Montes Corazao, profesora del curso de Psicolingüística en la Pontificia Universidad Católica del Perú. También es oportuno señalar nuestro agradecimiento a la sociolingüista Carolina Arrunátegui Matos por sus observaciones al presente manuscrito. 


\begin{abstract}
Mothers often speak to their children from an early age to stimulate their communication skills and the learning of their mother tongue. This special form of communication developed between mothers (and caregivers in general) and children is called baby talk. In the acquisition process, baby talk play a central role as providers of linguistic input. The objective of this study is to analyze the Peruvian baby talk. Methodologically, two mothers and their children in early childhood have been selected and their verbal interactions recorded. In this study, the linguistic characteristics and the styles of this baby talk are described. Likewise, the difference in speech between the receptive-style mother and the unreceptive-style mother is presented.
\end{abstract}

Key words: motherese, language acquisition, Spanish, style, communication.

Recibido $5 / 1 / 2020$

Aprobado 25/2/2020

\title{
1. Introducción
}

Cuando los niños adquieren su lengua, lo hacen de forma fácil, rápida y con un mínimo de esfuerzo. La adquisición se da de forma automática gracias a un conjunto de estructuras cognitivas universales e inherentes al ser humano que hacen a los niños capaces de construir su lenguaje sin instrucciones o entrenamiento específico (Muller Gathercole y Hoff, 2007, p. 109).

Aunque los padres u otros cuidadores no «enseñan» a los niños a hablar, juegan un rol importante en el proceso de adquisición al interactuar verbalmente con ellos. Un niño que no ha sido expuesto a ningún estímulo lingüístico no podrá desarrollar su lengua normalmente. De este modo, los padres o cuidadores constituyen una de las fuentes más importantes de input que el niño necesita para inducir las reglas de la gramática de su lengua (Mueller y Hoff, 2007). Es necesario señalar que el habla de los padres no es el único estímulo lingüístico que reciben los niños. Las conversaciones no dirigidas a él, pero que ocurren en su entorno, constituyen también input que el niño utilizará para construir su gramática.

El objetivo de este trabajo es analizar el maternés de dos madres peruanas. Para ello, se examinan las características lingüísticas, fonológicas, morfológicas, sintácticas, léxicas y pragmáticas, en la interacción lingüística madre-hijo. Además, se analizan los estilos de maternés que se dan en las interacciones. La importancia de este trabajo radica en presentar una descripción y explicación exploratoria sobre el habla de las madres en el Perú. Consideramos relevante realizar un examen de esta variedad lingüística poco estudiada (Moreira de Souza, Lidia y Abu-Jamra, 2010; Fernald, 1985; Liu, Tsao y Kuhl, 2009). 
El maternés de madres peruanas: Una descripción psicolingüística sobre...

El artículo se ordena de la siguiente manera: se presenta, a continuación, un marco conceptual; luego, se propone la metodología; de inmediato, se desarrolla el análisis; finalmente, se registran las conclusiones.

\section{El maternés y el entorno social}

\subsection{El maternés}

El input lingüístico que los niños reciben de sus padres o cuidadores recibe el nombre de maternés (Benítez, 2009, pp. 47). En la bibliografía, se le encuentra con diversos nombres: baby talk (Whyatt 1994), motherese (Pinker, 1995), madreleño (Bernárdez 1999), infant-directed speech (Thiesen, Hill y Saffran 2004). Incluso, se postula la denominación de paternés para el habla específica del padre hacia sus hijos. Si se trata del habla de los profesores en aula, se le conoce como teacher talk (Hitotuzi, 2005). El habla de los padres o cuidadores sirve para formular la hipótesis del habla materno (Rivero, 1993).

El maternés ha sido definido como el habla especial que utilizan los adultos cuando se dirigen a los niños. Al emplear el maternés, los adultos toman a los niños como interlocutores válidos, como si ellos entendieran lo que se les dice (Flores, Beltrami y De Souza, 2011); les adscriben también intenciones comunicativas e interpretan sus expresiones como voluntarias, conscientes e intencionales. En realidad, los adultos saben que los niños no entienden las palabras en sí mismas; no obstante, esta sobrevaloración de las competencias infantiles no constituye un obstáculo, sino, por el contrario, ayuda al niño a construir un sistema de signos al servicio de la comunicación (Rivero 1994). De este modo, la interacción se desarrolla por turnos entre el maternés del adulto cuidador y los llantos, gimoteos, risas y patadas del niño (Shiro, 2013).

El maternés se diferencia sustancialmente del habla dirigida a los adultos en distintos aspectos de su gramática. Diversos autores han descrito detalladamente sus características (Karmiloff y Karmiloff, 2001; Gallardo y Gallego, 200o; Whyatt, 1994; Rivero, 1994). Aquí listamos algunas de las más importantes:

a. En el plano fonológico:

- Pronunciación más cuidada

- Pausas más largas 
- Acentuación y contornos de entonación exagerados, especialmente en construcciones imperativas e interrogativas

\section{b. Sintaxis:}

- Cláusulas completas

- Oraciones cortas y simples

- Abundancia de construcciones imperativas e interrogativas

\section{c. Léxico y semántica}

- Se abordan temas que afectan al bebé aquí y ahora

- Léxico restringido

\section{d. Pragmática / conversación}

- Repeticiones de palabras

- Abundancia de vocativos

Como puede observarse, con el maternés, los adultos modulan y modifican su lenguaje para adaptarse a las necesidades del niño.

Cabe señalar que, en las alternancias de turnos entre la madre y el niño, ocurren balbuceos, sonrisas, llantos, quejidos y gestos. Los niños emplean lenguaje no verbal para interactuar con la madre y su entorno. Al respecto, las madres pueden usar esos llantos y quejidos para reforzar o reafirmar las actitudes o hechos del niño. Las madres tratan a sus hijos como a un participante de la comunicación.

\subsection{El entorno social: el habla receptiva y el habla poco receptiva} Bernstein (1971, citado por Gallardo y Gallego, 200o, pp. 91-92) señala que la procedencia social parece influir en las características del maternés de los adultos cuidadores y, por ende, en el proceso de adquisición. La forma en que se selecciona aquello que se considera relevante o foco de atención es distinta en función de las clases sociales. En un medio familiar de clase media o alta, el maternés de la madre está orientado a nombrar el mundo al niño, de modo que este desarrolla habilidades de adquisición léxica y, con ello, la capacidad de construir significados relativamente independientes del contexto (Hoff, 2005). Por otro lado, el maternés de las madres de un nivel socioeconómico bajo se orienta hacia significados relativamente ligados al contexto, de un universo restringido, muy poco estructurado. Esto da lugar a que el niño desarrolle un léxico ligado al contexto. 
El maternés de madres peruanas: Una descripción psicolingüística sobre...

Al respecto, Álvarez (2006) ha identificado dos estilos distintos de habla materna. El primero de ellos se denomina habla materna receptiva y, el segundo, habla materna poco receptiva. Ambos están vinculados con los dos estilos de adquisición: el estilo referencial y el estilo contextual (o expresivo). Los niños de madres de estilo receptivo tienen más características del estilo referencial, mientras que los niños de madres poco receptivas presentan características del estilo expresivo. Según Álvarez, las madres receptivas se caracterizan por hablar sobre objetos en los que el niño está interesado, aceptan las emisiones del niño, aunque procuran dar información correcta sobre dichas emisiones y están más dispuestas a responder cuando el niño solicita su atención. Por otro lado, las madres poco receptivas tienen un comportamiento más directivo, intentando imponer su foco de atención al niño y presentan una menor preocupación por sus respuestas verbales.

Ambos estilos se diferencian en dos aspectos fundamentales, los cuales se resumen a continuación.

Tabla 1. Estilos de habla de madres

\begin{tabular}{l|l|l}
\hline & Estilo receptivo & Estilo no receptivo \\
\hline Directividad & $\begin{array}{l}\text { La madre sigue el foco de atención } \\
\text { del niño. Habla sobre los objetos que } \\
\text { interesan al niño. }\end{array}$ & $\begin{array}{l}\text { La madre impone el foco de } \\
\text { atención al niño. }\end{array}$ \\
\hline Aprobación & $\begin{array}{l}\text { La madre acepta las emisiones y el } \\
\text { comportamiento del niño, aunque } \\
\text { pueda corregirlos luego. }\end{array}$ & $\begin{array}{l}\text { La madre se muestra } \\
\text { exigente y restrictiva. }\end{array}$ \\
\hline
\end{tabular}

Fuente: Adaptado de Álvarez (2006)

\section{Metodología}

Esta es una investigación cualitativa, descriptivo-explicativa, transversal. En esta, han participado dos parejas de madres con hijos peruanos, las cuales han aportado dos interacciones de media hora de duración cada una. Las edades de los niños son 7 meses y medio y 13 meses. Trabajar con niños en el último tercio del primer año responde a un determinado criterio de selección. Se considera que, en esta etapa, los bebés empiezan a mostrar la intención de comunicarse con el adulto, lo que 
vuelve más rica la interacción (Rivero 1994). Las interacciones fueron grabadas para su posterior trascripción y, al final de cada sesión, se administró una ficha sociolingüística que recoge los datos personales y familiares de los padres y los niños. La ficha tiene por objetivo recoger aquellos datos del entorno social que pudieran tener incidencia sobre las características del maternés.

Teniendo en cuenta las características del maternés, describiremos, en primera instancia, las semejanzas del habla materna según los siguientes niveles lingüísticos: fonología, morfología, sintaxis, léxico y pragmática. Luego, describiremos, la producción lingüística del maternés de cada madre entrevistada, clasificándola dentro de las nociones de madre receptiva y madre poco receptiva por presentarse una diferencia significativa entre ambas.

\section{Análisis}

Primero, se describen las características del maternés sobre la base de las dos interacciones madre-hijo (Madre A y Madre B) que integran nuestro corpus. Luego, se analizan los estilos de maternés.

\subsection{Descripción del maternés}

\subsubsection{Fonología}

En términos fonológicos, uno de los rasgos presentes en el maternés de las madres es el uso de la entonación marcada, casi exagerada, que es empleada para captar la atención de su niño. Esta prosodia dirige la actuación del niño para atender a lo que la madre describe o para realizar lo que la madre pide.

(1) MADRE A

¿Dónde está? ¿Se perdió? HII HII ¡SE PERDió EL PAPELITo! ¡No hay papelito! ¡Hijito! ¿mira! ¿el corazón! ¡AYY, QUÈ LINDO EL CORAZón! ¿Claudio! (...) ¿qué pasa? ¿Mmm? No los conocees ... ¿mmmm?

\section{(2) MADRE B}

Mira, mira ¿Quién está ALLííín?

Miiira ${ }_{i}$ No quieres ir al pasto a JUGAAAAR? 
El maternés de madres peruanas: Una descripción psicolingüística sobre...

Las madres marcan la entonación por lo general al final de los enunciados o en toda una frase clave. Asimismo, es característico de las madres el empleo del alargamiento de vocales, rasgo fonológico que producen para emitir afectividad y cariño a sus hijos.

(3) MADRE A

¡Bravooo!

¡Mira! Ahh, qué FreEscoo!

(4) MADRE B

¿Quiiieeres ir allaaá a jugar pelota!

¡Uuuyyyy que bonito, que boniiito ese bebeeeé!

Fonológicamente, las madres adaptan su lenguaje a un estilo infantil de habla para mostrar cariño a sus bebés. Así, ocurren cambios o elisiones consonánticas en las palabras.

(5) MADRE A

¿Qué «cocha»?

¿Qué «pacha»?

¡qué «LICO»!

(6) MADRE B

Ahí tá

En el acoplamiento del habla materna al infantil, suele observarse juegos verbales silábicos, los cuales tienen como función estimular el estado anímico del bebé.

(7) MADRE A

¡BRAVOoo! ¡Claudio! ¡Bravo! (mientras el niño aplaude)

¡Esos CINcooo! ¡Esos CINcooo! (mientras el niño y la madre chocan las palmas de las manos)

EESo, eso, eso, EESo, eso, eso, eso, eso, EEso, eso, eso, eso, EESo (mientras la madre hace saltar al niño sosteniéndolo de los brazos)

¡Qué-pena-qué-pena qué-pena-qué-pena-qué-penaaa! (mientras la madre hace saltar al niño sosteniéndolo de los brazos) 


\subsubsection{Morfología}

Uno de los rasgos morfológicos extendidos en el habla peruana es el uso del diminutivo, el cual se ha visto ligado al habla afectiva o amical, pero al cual se lo ha relacionado con el habla materna. En este sentido, consideramos que el empleo de diminutivos es una característica del maternés, pues las madres transmiten, a través de su uso, una carga valorativa y apreciativa de lo que enuncia y comunica al bebé.

\section{(8) MADRE A}

Toma tu bolsita, la bolsita.

Vasitos No! ¡Vasito no!

Bolita, busca bolita.

\section{（9） MADRE B}

¿Quieres una galletita?

Tienes que agarrarte, agáchate un poquito, pasa, pasa

Vamos al otro pasto, ahí, ahí un ratito si quieres

\subsubsection{Sintaxis}

Sintácticamente, el maternés se caracteriza por una gramática simplificada, como el uso de frases cortas y directas.

(10) MADRE A

(señala el balde) ¡Ponlo aquí!

¡Tus galletas!

¡Vasitos No! ¿Vasito No!

\section{(11) MADRE B}

Vamos, ven, vamos, mira

No obstante, la sintaxis no es absolutamente reducida, sino que hay presencia de construcciones extensas y ricas en la descripción que hace la madre sobre el mundo para el bebé. En este contexto, aparecen construcciones oracionales completas. 
El maternés de madres peruanas: Una descripción psicolingüística sobre...

(12) MADRE A

¿Bravo? ¡Bravo! Pero «bravo» no se puede hacer bravo con bolsa

Ah, ahí está la estrella

A ver, te quito las medias...

(13) MADRE B

Ahí tá, ese te gustó, ese es lo que te gustó y este de aquí, este, no, no, este te he dicho, este no, quieres este, este sí

Mira Luis Alberto aquí hay una galletita, ven párate, párate...

Al respecto, es notorio encontrar en el maternés el empleo de interrogativas con sentidos completos. Este rasgo sintáctico se usa para dialogar con el bebé, se busca respuestas dentro de la interacción madre-niño.

(14) MADRE A

¿Estás molesto?

¿Estás cansado? ¿Estás de mal humor?

¿Tás con sueño? ¿Con calor ya?

¿Te hago cosquillas ahí en tu PIECITo?

¿Qué quieres?

¡¿Qué pasa hijo?!

(15) MADRE B

Uuuuyyy ¿Qué pasó?

Ya mira, mira, ¿quieres ir?

Vamos, allá está Juan Jesús ¿Quieres ir a verlo?

También es muy recurrente la aparición de exclamaciones de frases nominales que se utilizan para establecer y mantener el contacto comunicativo con los niños.

(16) MADRE A

¡Holaa! Holaa!

¡Tus galletas!

¡El corazón!

¡Piecitos! 
(17) MADRE B

¡Uuuuy qué boniiiito!

Y otra característica sintáctica singular del maternés es el uso de imperativas para dirigir el accionar de los bebés. Las madres suelen emplear imperativas constantemente, porque están pendientes de la conducta y comportamiento de los niños. La advertencia es uno de los aspectos que sobresalen.

(18) MADRE A

Toma tu bolsita, la bolsita

Tápalas. Tápalas. Tápalas. A ver, las galletitas, tápalas

(19) MADRE B

Vamos por allá ven, vamos por aquí

No, no se agarra, pues no se agarra, no se agarra ... pasa corriente No, no puedes entrar

Dame la galleta

Párate, párate, Luis Alberto

\subsubsection{Léxico}

En términos léxicos, aunque las madres presentan un registro léxico como hablantes de una lengua, es peculiar encontrar voces léxicas pertenecientes a un registro maternal. Este registro aparece en el contexto madre-niño, constituyéndose, de esta manera, el maternés como una variación situacional. Estas voces se relacionan con el mundo infantil. Las madres usan palabras que sirvan para integrar a su hijo a un clima familiar e infantil.

(20) MADRE A

Ven chiquitín. Tienes sueño

(21) MADRE B

Miiiira, uuuuyyyy otro bebeee

Las misses de kinder tienen juguetitos ahí 
El maternés de madres peruanas: Una descripción psicolingüística sobre...

En el contexto lexical sobre el maternés, suele aparecer el reemplazo de nombres de cosas por nombres que resultan más cercanos o familiares para el niño. La madre hace sustituciones léxicas para orientar la capacidad mental del niño para categorizar la información conceptual.

\section{(22) MADRE A}

¡No! Bolita busca, bolita. (La madre le acerca un adorno esférico al niño, que no es una bola)

A ver, las galletitas, tápalas (La madre señala el balde de juguete. La madre emplea el término "galletitas» para hacer referencia al balde de juguete donde hubo antes galletas)

\subsubsection{Pragmática}

La relación de la madre con el bebé genera diálogos y tomas de turno que muestran la interacción social dada entre ambos. La interacción discursiva desemboca en la atención que se brinda al niño como foco de la adquisición lingüística.

(23) MADRE A

¡Qué feo que es esto! (La madre retira el estuche del CD)

(El niño se da cuenta)

¿Qué, qué?

(El niño se incomoda y se lamenta lloriqueando)

¡Nooo, no Llores!

\section{(24) MADRE B}

No, no se agarra, pues no se agarra, Luis Alberto, no se agarra, pasa corriente

(Llora)

Ahhh, no vas a agarrar eso, ahh, noo... no no botes, tú eres bien vivo ¿no?

En términos pragmáticos, hay presencia de redundancia de expresiones lingüísticas para enfatizar algún elemento comunicativo y ganar más expresión e intención comunicativa. Las repeticiones son una estrategia del discurso que muestra 
las intenciones comunicativas expresivas de los hablantes al emplear la repetición en el discurso oral (Poblete, 2002).

(25) MADRE A

¿Esos CINCOO, esos CINCOo!

Vasitos No! ¡Vasito no!

EESo, eso, eso, EEso, eso, eso, eso, eso, EEso, eso, eso, eso, EESo (la madre hace saltar al niño tomándolo de las axilas)

(26) MADRE B

Vamos al otro pasto, al otro pasto

Te chapo, te chapo, te chapo. Despacio

Cuidado, cuidado, despacio, despacio que te caes, dame eso, dame eso

Por último, otro rasgo pragmático es la utilización del vocativo para evidenciar que se está dirigiendo al bebé dentro de la interacción.

(27) MADRE A

¿BRAvoo, BRAvooo! Claudio, ¿los cinco?

Claudio, bravo pu no dices NADA!

(28) MADRE B

A ver mira. Pesas, Luis Alberto ¿Ahí si entras, Luis Alberto, no?

Mira allá arriba, mira, Luis Alberto, aquí mira, mira aquí

Todos estos rasgos, desde el nivel fonológico hasta el pragmático, son los que han aparecido en las dos madres entrevistadas. Estos rasgos se tornan como rasgos similares entre los dos maternés y el maternés como una noción casi universal. 
El maternés de madres peruanas: Una descripción psicolingüística sobre...

\subsection{Estilos de maternés}

\subsubsection{Directividad}

La directividad está referida a quién dirige el tema de la interacción, estableciendo qué es lo relevante o cuál es el foco de atención. Esta característica es propia del maternés de estilo poco receptivo. Con respecto a nuestra muestra de interacciones, hemos encontrado que el maternés de la Madre A (Interacción 1) se muestra bajo en directividad; en este caso, la madre está todo el tiempo pendiente de qué es lo que desea el niño o qué es lo que está mirando:

(29)

MADRE A: ¿Qué? ¿Qué quieres? ¿Esto?

(El niño suspira)

MADRE A: Ya. Esto. (Acerca la envoltura de plástico al niño).

(30)

MADRE A: ¡Claudio! ¿Qué pasa hijo? ¡Ahhh! ¿Estás cansado? ¿Estás de mal humor? ¿Estás molesto? ¿La estrella? (le alcanza al niño una pieza de plástico en forma de estrella).

(31)

(El niño empieza a hacer sonidos repetitivos: he-he-he-he-he)

MADRE A: Ah, ha visto el celular. A ver, presta (al que filma).

Por otro lado, la Madre B muestra un comportamiento directivo en tanto señala al niño lo que debe mirar y a qué debe prestar atención en múltiples ocasiones:

(32)

MADRE B: Ahí está, allí está el ventilador, Luis Alberto, mira, mira allá arriba, mira, Luis Alberto, aquí, mira aquí.

(33)

MADRE B: Mira aquí, Mono (mientras le señala la imagen de un mono) no ACÁ, no sé qué es este, este es tortuga de mar, tampoco sé que es 
este, este es un tigre, el oso panda, otro mono, elefante, tigre, oso. Ahí 'ta, ese te gustó, ese es lo que te gustó y este de aquí, este, no, no, este te he dicho, este, no quieres este, este sí, este, este también.

\subsubsection{Aprobación}

El criterio de aprobación se refiere al grado de aceptación que muestra la madre respecto de las emisiones o del comportamiento de su hijo. La Madre A presenta un comportamiento bastante aprobatorio, respondiendo con entusiasmo al comportamiento de su hijo:

(34)

MADRE A: Claudio, ¿los cinco? (enseña la palma de la mano al niño) ¡Esos Cincooo! ¡Esos cincooo! (después que el niño le da los «cinco» a la madre) ahiahiahi... ¡Esos CINcooo! (palmotea la mano del niño). ¡BRAVOoo! BRAVOoo!

(35)

(La madre observa al niño jugar con su juguete, un balde de plástico. El niño logra sacar la tapa del balde de plástico).

MADRE A: ¡BRAVOoo! BRAVOoo!

La Madre B, por su parte, se muestra pocas veces entusiasta con las iniciativas de su hijo; por el contrario, hay una tendencia a desaprobar su conducta.

(36)

MADRE B: Mira, pero dame la galleta, dame la galleta, ¿no quieres?, toma el celular, toma, toma. Quieres agarrar todo, dame la galleta. No quieres soltar la galleta.

(37)

MADRE B: No puedes entrar; además, dice que no te saques el zapato. 
El maternés de madres peruanas: Una descripción psicolingüística sobre...

\subsection{Presencia de construcciones imperativas}

Se pudo observar una diferencia notable entre las madres respecto del uso de construcciones imperativas en sus interacciones. La Madre B presentó un promedio de 95 estructuras imperativas, mientras que la Madre A presentó 43. Creemos que la abundancia de formas imperativas podría pertenecer al estilo de maternés poco receptivo, en tanto es un indicador de que la madre no está pendiente de los intereses de su niño, sino que, por el contrario, antepone los suyos a los de él:

$(38)$

MADRE B: Ahí nomás Luis Alberto, ahí nomás, hijo. PÁrate, PÁrate, PÁrate. Luis Alberto, ven para acà, ahorita te van a patear.

(39)

MADRE B: Cuidado te caes, cuidado te caes. Luis Alberto ven, cuidado te caes, vEN Luis Alberto, no, no puedes entrar, NO, no puedes entrar, ven por acá, vamos por allà, ven por aquí.

La Madre A, por su parte, hizo también uso de este tipo de estructuras, pero casi siempre incluyó un diminutivo con la intención de suavizar el tono imperativo:

(40)

MADRE A: ¡Papelito no!

$(41)$

MADRE A: A ver, las galletitas, tápalas, ponlo aquí.

(42)

MADRE A: No, vasitos, no, vasitos no. No, hijito.

\section{Conclusiones}

Las características distintivas del maternés están presentes en las interacciones de nuestro corpus. El maternés de ambas madres peruanas presentan rasgos de prosodia (uso de entonación marcada), morfología (uso de diminutivo), sintaxis 
(producción de frases cortas y largas, con tipos de cláusulas interrogativas e imperativas), léxico (léxico relacionado con el mundo infantil), pragmática (interacciones discursivas y uso de vocativo). En la atención que las madres ponen en sus niños, las madres buscan la interacción comunicativa con sus hijos y tratan de facilitarles la comprensión de los contenidos que intentan trasmitir; por ello, echan mano de su habla materna. En este sentido, hay ciertas convergencias o semejanzas en los estilos de la madre o maternés empleado. Esto nos indica que el maternés más que una lengua es una variación lingüística sometida al acto comunicativo con el niño. Algunas características extralingüísticas notoria, aunque no estudiadas con detenimiento, presentes en el maternés, han sido los gestos, las mímicas, las risas y los llantos que forman parte importante de la comunicación.

Los estilos de maternés de madre receptiva y no receptiva están presentes en las interacciones lingüísticas madre-hijo que forman parte de nuestro corpus. Consideramos que factores sociales como el estrato socioeconómico de las madres influencian la aparición de un estilo u otro. Siguiendo a Berstein (1971), creemos que entornos familiares de clase media ofrecen un estímulo más rico (estilo receptivo) para el desarrollo lingüístico del niño, que aquel que ofrecen entornos familiares de clase baja (estilo poco receptivo). Esto puede corroborarse con los datos de nuestra ficha sociolingüística: la Madre B pertenece a un estrato socioeconómico bajo, mientras que la Madre A pertenece a uno medio. Esto podría explicar el estilo de maternés que cada una presenta en sus interacciones. Otro factor que podría también tener incidencia en el tipo de maternés adoptado es el número de hijo que es el niño de cada interacción. En el caso de la Madre A, el niño es hijo único; en el caso de la madre B, el niño es su cuarto hijo. Muy probablemente, el estilo de la madre A sea más receptivo que el de la $\mathrm{B}$ porque su atención y sus cuidados están enfocados en su único hijo, a diferencia de la Madre B, que debe cuidar de tres niños más.

Cabe señalar que los factores psicolingüísticos y sociolingüísticos pueden variar en contextos específicos diferentes (Ibáñez 2000). Sus incidencias en la adquisición pueden ser disímiles (Montes y Lovón 2014). Las conclusiones a las que arribamos no son necesariamente absolutas. Se requieren realizar trabajos más amplios, por ejemplo, sería importante estudiar la interacción entre madre e hijas, padres con hijos e hijas, niñeros y niños, abuelos y nietos.

Asimismo, es necesario señalar que hay investigación reciente (Blanco, 2005) que, desde una mirada crítica hacia el variacionismo lingüístico y los estudios correlacionales en general, cuestionan las asociaciones muchas veces esencialistas 
El maternés de madres peruanas: Una descripción psicolingüística sobre...

que se establecen entre formas de hablar o de utilizar el lenguaje y variables sociales como clase, educación, género, etc. En ese sentido, debemos concebir la presente investigación como un estudio inicial que necesita ser articulado en el futuro con estas otras miradas contemporáneas, las cuales pueden ser iluminadoras con respecto a las relaciones entre el maternés y el entorno social. En relación con ello, se precisa que el estrato social no puede ser el único factor determinante para los estilos de habla, pues hay diversos factores que afectan el uso. Nuevos estudios podrán estudiar el contexto social más allá de la propuesta la clase socioeconómica que hemos manejado y, por tanto, invitamos a realizarlos.

\section{Referencias bibliográficas}

Álvarez, S. (2006). Relación entre el habla materna y la adquisición y desarrollo del lenguaje en el niño. MAPRE Medicina, 17 (3), 202-215. Recuperado de http:// sid.usal.es/idocs/F8/ART9362/relacion.pdf

Benítez, A. (2009). Genes y lenguaje. Aspectos ontogenéticos, filogenéticos y cognitivos. Barcelona: Reverté.

Bernárdez, E. (1999). ¿Para qué sirve el lenguaje? En Autor, ¿Qué son las lenguas? (cap. 9). Madrid, Alianza.

Blanco, C. (2005). Sociolingüística y análisis del discurso: herramientas para la investigación en educación. Revista de Pedagogía, 26(76), 307-352. Recuperado de http://ve.scielo.org/scielo.php?script=sci_arttext\&pid=So798 97922005000200006\&lng=es\&tlng=es

Clemente, E., y Remírez, J. (2000). Relación entre el habla maternal y la infantile en una tarea de interacción conjunta. Revista de Psicología General y Aplicada, 53(2), 387-400. Recuperado de http://dialnet.unirioja.es/servlet/ articulo? codigo $=2356962$

Fernald, A. (1985). Four-Month-Old Infants Prefer to Listen to Motherese. Infant behavior and development, 8, 181-195. Recuperado de http://www-psych.stanford.edu/ babylab/pdfs/Fernald\%201985.pdf

Flores, M., Beltrami, L., y De Souza, A. (2011). O manhês e suas implicações para a constituição do sujeito na linguagem. Disturbios da Comunicação, 23(2), 143-152. Recuperado de https://revistas.pucsp.br/dic/article/view/8270

Gallardo, J., y Gallego, J. (2000). Manual de logopedia escolar. Un enfoque práctico. España: Aljibe. 
Hitotuzi, N. (2005). Teacher Talking Time in the EfL Classroom. Profile Issues in Teachers' Professional Development, 6, 97-106. Recuperado de https://revistas. unal.edu.co/index.php/profile/article/view/11129

Liu, H.-M., Tsao, F.-M., y Kuhl, P. K. (2009). Maternal Speech to Infants in Mandarin Chinese: Support for an Expanded Vowel Triangle in Mandarin Motherese. Washington: University of Washington.

Ibáñez, N. (2000). El lenguaje en el niño: una nueva mirada. Estudios pedagógicos, 26, 51-67. https://dx.doi.org/10.4067/So718-07052000000100004

Karmiloff, K., y Karmiloff, A. (2001). Pathways to Language: From Fetus to Adolescent. Cambridge: Harvard University Press.

Montes, C., y Lovón, M. (2014). La adquisición de una segunda lengua Reflexiones teórico-prácticas. Lima: Facultad de Educación de la PUCP.

Mueller, V., y Hoff, E. (2007). Input and the Acquisition of Language: Three questions. En E. Hoff, y M. Shatz (ed.), The Handbook of Language Development (pp. 107-127). Blackwell. https://doi.org/10.1002/9780470757833.ch6

Moreira de Souza, M., Levy, L., y Abu-Jamra, S. (2010). O manhês: costurando laços. Estilos da Clinica, 15(2), 420-433. Recuperado de http://www.revistas. usp.br/estic/article/view/46099

Pinker, S. (1995). El instinto del lenguaje. Cómo crea el lenguaje la mente. Madrid. Alianza.

Poblete, M. T. (2002). Las repeticiones en la interacción comunicativa. Revista de Lingüística Teórica y Aplicada, 40, 147-170. Recuperado de https://dialnet. unirioja.es/servlet/autor? codigo $=16159$

Rivero, M. (1993). La influencia del habla de estilo materno en la adquisición del lenguaje: valor y límites de la hipótesis del input. Anuario de Psicología, 57, 45-64. Recuperado de https://dialnet.unirioja.es/servlet/articulo? codigo $=2946510$

Shiro, M. (2013). Expresiones de afecto de madres bilingües. Actualidades en Psicología, 27(115), 75-91. https://doi.org/10.15517/ap.v27i115.8991

Thiesen, E., Hill, E., y Saffran, J. (2004). Infant-Directed Speech Facilitates Word Segmentation. Infancy, 7(1), 53-71. http://dx.doi.org/10.1207/ S15327078ino701_5

Whyatt, B. (1994). Baby talk. The Language Addresed to Language-Acquiring Children: A review of the Problem. Studia Anglica Posnaniensia, 29,125-135. Recuperado de http://ifa.amu.edu.pl/sap/files/29/10Whyatt.pdf 